\title{
RESGATE DE MUDAS DE Lychnophora pohlii COMO ALTERNATIVA PARA RECUPERAÇÃO E CONSERVAÇÃO DE CAMPO RUPESTRE
}

\author{
Nathália Ferreira e Silva ${ }^{1}$, Israel Marinho Pereira ${ }^{2 *}$, Miranda Titon ${ }^{2}$, Márcio Leles Romarco de Oliveira $^{2}$, \\ Marcelo Luiz Laia ${ }^{2}$, Luana Cristielle Araújo ${ }^{3}$ \\ ${ }^{1}$ Cedrus Consultoria e Soluções Ambientais Ltda., Divinópolis, Minas Gerais, Brasil - nathfs7 @ hotmail.com \\ ${ }^{2 *}$ Universidade Federal dos Vales do Jequitinhonha e Mucuri, Departamento de Engenharia Florestal, Diamantina, Minas Gerais, \\ Brasil - imarinhopereira@gmail.com; titonmiranda@yahoo.com.br; marcioromarco@gmail.com; marcelolaia@gmail.com \\ ${ }^{3}$ Universidade Federal dos Vales do Jequitinhonha e Mucuri, Programa de Pós-Graduação em Ciência Florestal, Diamantina, Minas \\ Gerais, Brasil - luaninhaaraujo_engflorestal@hotmail.com
}

Recebido para publicação: 17/05/2013 - Aceito para publicação: 10/03/2015

\begin{abstract}
O objetivo deste trabalho foi avaliar o efeito do tamanho da planta resgatada e dos níveis de redução foliar na sobrevivência, crescimento e emissão de folhas em mudas de arnica obtidas via resgate em um remanescente de campo rupestre. Foram resgatados 240 indivíduos, os quais foram divididos em duas classes de altura (Classe I-2,5 a $20 \mathrm{~cm}$ e Classe II -25 a $55 \mathrm{~cm}$ ) e submetidos a três intensidades de redução foliar $(0 \%, 50 \%$ e $100 \%)$. As medições de altura, diâmetro e emissão de novas folhas foram realizadas em nove tempos $(0,15,30,45,60,75,90,105$ e 120 dias $)$ e a avaliação da sobrevivência aos 120 dias. A taxa média de sobrevivência foi de 49,2\%, sendo maior na Classe I e não apresentando diferença estatística quanto aos três tipos de redução foliar. A emissão de folhas foi maior nos indivíduos que sofreram redução foliar, observando-se uma diminuição ao longo do tempo, ao contrário das mudas com $0 \%$ de redução, que tiveram um crescimento linear. Portanto, é aconselhável que o resgate de mudas de arnica seja realizado para plantas com tamanho entre 2,5 e $20 \mathrm{~cm}$, sem necessidade de redução foliar.
\end{abstract}

Resumo

Palavra-chave: Lychnophora pohlii; produção de mudas; redução foliar; classe de altura.

\begin{abstract}
Evaluation of the arnica plant rescue as an alternative to conservation and restoration of campo rupestre ecosystems. The objective of this research was to evaluate the effect of rescued plant size and levels of reduction in leaf survival, growth, and insertion of leaves in seedlings of Arnica obtained via salvage in a remnant of Campo Rupestre. We rescued 240 individuals and these were divided into two classes' height (Class I -2.5 to $20 \mathrm{~cm}$ and Class II $-25-55 \mathrm{~cm}$ ) and subjected to three levels of reduction leaf $(0 \%, 50 \%$ and $100 \%)$. The measurements of height and diameter and new leaves emission were collected at nine times $(0,15,30,45,60,75,90,105$ and 120), and evaluation of survival at 120 days. The average survival rate was $49.2 \%$, higher in Class I and had no significant difference regarding the three types of leaf reduction. The emission sheet was higher in subjects experiencing a leaf reduction observing a decrease over time, unlike the seedling with $0 \%$ reduction, which increased linearly. Therefore, it is advisable a size between 2.5 and $20 \mathrm{~cm}$ for the rescue of arnica seedling plant, without leaf reduction.
\end{abstract}

Keywords: Lychnophora pohlii; seedlings production; leaf reduction; height class.

\section{INTRODUÇÃO}

Dentre as espécies endêmicas dos campos rupestres que se encontram em áreas de distribuição restrita, destaca-se Lychnophora pohlii Schultz-Bip, espécie que possui grande importância ecológica e econômica e se encontra na situação "em perigo (EN)" na lista vermelha da flora ameaçada do Brasil (MARTINELLI; MORAES, 2013). É conhecida popularmente como arnica e pertence à família Asteraceae, sendo amplamente utilizada na medicina, tanto popular quanto científica, e indicada para recuperação de áreas degradadas (GRAEL et al., 2004; KANASHIRO et al., 2006; JACOBI et al., 2008; VENDRUSCOLO; MENTZ, 2006; HEIDEN et al., 2007).

FLORESTA, Curitiba, PR, v. 45, n. 3, p. 645 - 654, jul. / set. 2015.

Silva, N. F. e. et al.

ISSN eletrônico 1982-4688 / ISSN impresso 0015-3826

DOI: $10.5380 /$ rf.v45i3.31949 
Segundo Heiden et al. (2007), a eficiência na dispersão confere às plantas da família Asteraceae extrema importância no conhecimento da recuperação de áreas degradadas, onde participam tanto como pioneiras quanto na ocorrência em clareiras e bordas de mata na colonização de ambientes degradados, porém algumas espécies dessa família não se comportam dessa maneira (Melo et al., 2007).

Jacobi et al. (2007), em levantamentos florísticos em duas áreas de campos rupestres ferruginosos no Quadrilátero Ferrífero, uma na Serra do Rola Moça e outra na Serra da Moeda, observaram que a arnica é uma das espécies mais comuns. Sua alta sobrevivência nesses locais pode estar relacionada às adaptações morfológicas e fisiológicas, que favorecem o desenvolvimento nesses ambientes com grandes afloramentos rochosos (GIANOTTI et al., 2013). No entanto, devido a sua grande dificuldade de propagação, a exploração de seus recursos genéticos é obtida através de coleta extrativista extensiva (RODRIGUES; CARVALHO, 2001; SOUZA et al., 2003), muito impactante para as populações. Dessa forma, a propagação ex situ da espécie e o resgate de plantas jovens pode minimizar tais problemas e assegurar a perpetuação da espécie em seu hábitat natural.

O resgate de indivíduos da flora em áreas liberadas para supressão em empreendimentos minerários seria uma das opções. Segundo García-Orth e Martínez-Ramos (2011), tal prática é promissora para superar barreiras ambientais (ambientes em condições estressantes e disponibilidade de propágulos escassos), que afetam a sobrevivência, crescimento e reprodução de plantas em regeneração.

O transplante de mudas é mais vantajoso que a utilização de sementes por não apresentar tão alto risco de mortalidade e dispensar etapas tradicionais na produção de sementes (MARTINEZ-GARZA et al., 2005). Além disso, proporciona vários benefícios ecológicos, como disponibilidade de poleiros para dispersores, aumentando, assim, a chuva de sementes em campo aberto (HOLL, 2002), e promove a viabilidade de plantas ameaçadas de extinção (LAWRENCE; KAYE, 2011; CALEGARI, et al., 2011), além de facilitar o processo de sucessão.

Os poucos estudos existentes na literatura científica sobre resgate de plântulas jovens de espécies tropicais evidenciam a necessidade de redução de $50 \%$ das folhas de cada indivíduo (VIANI; RODRIGUES, 2007; CALEGARI et al., 2011; RIBEIRO et al., 2011). Esse processo auxilia no sucesso do estabelecimento de plântulas, devido à redução do estresse hídrico, e facilita a visualização da emissão de novas folhas pela planta. A redução do número de folhas visa evitar o efeito "guarda-chuva", que, potencialmente, prejudica a eficiência da irrigação, além de evitar a transpiração em excesso (SANTANA et al., 2010).

Os estudos sobre resgate de plântulas de espécies tropicais são escassos, mas necessários para preservação da biodiversidade, tanto que tem sido uma das principais condições impostas pelos órgãos ambientais no licenciamento de empreendimentos minerários e hidrelétricos. Assim, o objetivo deste trabalho foi obter informações sobre a eficácia da técnica de cultivo de plantas jovens de arnica (Lychnophora pohlii).

\section{MATERIAL E MÉTODOS}

O resgate de mudas de arnica (Lychnophora pohlii) foi realizado em áreas de campo rupestre, destinadas a supressão da vegetação para ampliação do Campus Juscelino Kubitschek, da Universidade Federal dos Vales do Jequitinhonha e Mucuri (UFVJM) em Diamantina, MG. O regime climático da região é tropical típico, Cwb na classificação de Köppen, caracterizado por verões brandos e úmidos e invernos mais frescos e secos. As médias anuais de precipitação variam entre 1250 e $1550 \mathrm{~mm}$, com temperaturas entre 18 e $19{ }^{\circ} \mathrm{C}$. A umidade relativa do ar se situa em torno de 75,6\% (NEVES et al., 2005).

Após estabelecer a área de coleta, a metodologia do resgate de mudas de arnica obedeceu à seguinte sequência:

a) Coleta: em dezembro de 2010, foram coletadas ao acaso, de forma cautelosa, para não causar danos às raízes, 240 plantas de arnica (120 da Classe I - 2,5 a $20 \mathrm{~cm}$ de altura e 120 da Classe II - 25 a $55 \mathrm{~cm}$ de altura), aparentemente sadias e sem sinal de injúrias. O material experimental foi destorroado manualmente na região das raízes e acondicionado imediatamente em recipientes contendo água.

b) Transporte: após o acondicionamento, as mudas foram conduzidas ao Centro Integrado de Propagação de Espécies Florestais (CIPEF), do Departamento de Engenharia Florestal da UFVJM, em Diamantina, MG, onde foi instalado o experimento imediatamente após a coleta. O CIPEF situa-se a aproximadamente $1390 \mathrm{~m}$ de altitude, sendo a precipitação e a temperatura médias anuais de $1404 \mathrm{~mm} \mathrm{e} 18,1^{\circ} \mathrm{C}$, respectivamente.

c) Transplante: as mudas foram transplantadas para sacos de polietileno $(30 \times 20 \mathrm{~cm})$ preenchidos com 
substrato composto por $70 \%$ de terra de subsolo (Neossolo), 30\% de Bioplant ${ }^{\circledR}$ e $7 \mathrm{~g} / \mathrm{L}$ de osmocote 15 9-12. As raízes excessivamente grandes ou tortas foram podadas quando excediam o recipiente de transplante.

d) Tratos culturais no viveiro: as mudas foram mantidas em casa de sombra coberta com sombrite de $50 \%$ de redução de luminosidade e irrigação por aspersão com vazão de $85 \mathrm{~L} / \mathrm{h}$. Após 100 dias, as mudas foram submetidas a um processo de rustificação a céu aberto e irrigação por 110 dias por aspersão com vazão de $200 \mathrm{~L} / \mathrm{h}$, até o final do experimento. O controle das ervas daninhas ocorreu periodicamente, de forma manual.

O experimento consistiu na avaliação de quatro características: crescimento (altura e diâmetro do colo), sobrevivência e emissão de folhas, de modo a identificar as respostas das mudas de arnica após a intervenção dos tratamentos. Para as duas primeiras características, foi utilizado o delineamento experimental inteiramente casualizado, com quatro repetições, no esquema de parcelas subdivididas $2 \times 3 \times 9$, sendo estudado na parcela principal o efeito de duas classes de tamanho (Classe I - 2,5 a $20 \mathrm{~cm}$ e Classe II - 25 a $55 \mathrm{~cm})$; na primeira subparcelas, três intensidades de redução foliar $(0 \%$ - sem redução foliar, $50 \%$ - redução em $50 \%$ de todas as folhas e $100 \%$ - remoção total das folhas); e na última, nove tempos $(0,15,30,45,60,75,90,105$ e 120 dias). A unidade experimental foi constituída por 10 mudas. Para a avaliação da sobrevivência, a única diferença em relação ao procedimento anterior foi a adoção apenas de tratamentos relacionados às classes de tamanho e redução foliar aos 120 dias, constituindo assim um esquema fatorial $2 \times 3$ (duas classes e três intensidades de redução). A emissão de folhas foi avaliada pela porcentagem de indivíduos que emitiram novas folhas ao longo dos tempos descritos, consistindo, desse modo, numa análise de regressão em função do tempo para cada combinação (classes x intensidade de redução), totalizando seis ajustes.

A altura total $(\mathrm{cm})$ foi definida como a distância do nível do substrato até a inserção da última gema e foi mensurada com auxílio de uma régua graduada. O diâmetro do colo $(\mathrm{mm})$ foi medido com um paquímetro digital. A porcentagem de sobrevivência (\%) foi calculada a partir do número de indivíduos mortos aos 120 dias, que se baseou na ausência de folhas e caules secos.

Os dados qualitativos foram submetidos à ANOVA e foi realizado o teste de média de Tukey a 95\% de probabilidade. Para o parâmetro tempo (t) (caráter quantitativo), realizou-se uma análise de regressão pelo método dos mínimos quadrados ordinários, em que o tempo foi a variável explicativa e altura total, diâmetro do colo e emissão de folhas foram as variáveis dependentes. A análise estatística foi realizada com auxílio do programa Statistica 10.0 (STATSOFT, 2010).

\section{RESULTADOS E DISCUSSÃO}

Verifica-se, na tabela 1 , que houve diferença significativa $(p<0,05)$ para redução foliar e interação tempo x classe para a característica altura, enquanto que para o diâmetro do colo a significância $(p<0,05)$ ocorreu para as classes e para o tempo. A significância estatística para a classe já era esperada, uma vez que as mudas de arnica foram separadas em duas categorias de alturas.

Para a variável altura, as intensidades de $50 \%$ e $100 \%$ de redução foliar foram estatisticamente diferentes entre si, mas sem diferença estatística quando comparadas à intensidade de $0 \%$ (Figura 1). Santana et al. (2010), ao estudarem oito híbridos de Eucalyptus urophylla e quatro níveis de redução (0\%, $25 \%, 50 \%$ e $75 \%$ ), verificaram que, para a variável altura, qualquer nível de redução foliar pode ser adotado, porém, de acordo com Carneiro (1995), isso é válido desde que as mudas estejam no intervalo de $15-35 \mathrm{~cm}$ de altura, definido como sendo o adequado para mudas de E. uroplylla. Já para a variável diâmetro do colo, $50 \%$ dos clones avaliados demonstraram efeito negativo com a redução foliar. Portanto, a não utilização da redução foliar foi a alternativa mais viável para a maioria dos clones avaliados.

Foi verificada ao longo do tempo uma elevada taxa de mortalidade nas plantas de maior porte. Desse modo, para não afetar as avaliações de crescimento em diâmetro do colo e altura em todas as medições, inclusive na primeira, foram consideradas apenas as plantas que sobreviveram até a última medição, ou seja, foi calculado o crescimento durante os 120 dias somente das 118 mudas sobreviventes. Foram realizados os ajustes com o modelo quadrático para altura da interação (classe $\mathrm{x}$ tempo) e com o modelo linear para o diâmetro em função do tempo.

A partir da segunda medição, observou-se que as mudas começaram a apresentar um leve decréscimo em altura, no entanto, cerca de 60 dias após, foi verificada uma recuperação das mesmas. Para o diâmetro do colo, foi observado um crescimento linear e positivo (Figura 2). Neste trabalho, foi constatado, durante os 60 dias iniciais, que os indivíduos de arnica murcharam e consequentemente perderam suas folhas, porém não deram indícios de estarem mortos.

FLORESTA, Curitiba, PR, v. 45, n. 3, p. 645 - 654, jul. / set. 2015.

Silva, N. F. e. et al.

ISSN eletrônico 1982-4688 / ISSN impresso 0015-3826

DOI: $10.5380 /$ rf.v45i3.31949 
Tabela 1. Resumo da análise de variância para as características altura total e diâmetro do colo das mudas de arnica ao longo dos 120 dias após o resgate.

Table 1. Summary of analysis of variance for full height features and diameter of arnica seedlings along the 120 days after the rescue.

\begin{tabular}{|c|c|c|}
\hline \multirow{2}{*}{ Fonte de variação } & Altura total $(\mathbf{c m})$ & Diâmetro do colo (mm) \\
\hline & $\mathbf{Q M}$ & QM \\
\hline Classes (c) & $28034,01 *$ & $526,12 *$ \\
\hline Resíduo a & 157,01 & 59,51 \\
\hline $\mathrm{CV}_{\exp }(\%)$ & 51,76 & 46,43 \\
\hline Reduções (r) & $436,17 *$ & $53,76^{\mathrm{ns}}$ \\
\hline $\mathrm{c} \times \mathrm{r}$ & $91,54^{\mathrm{ns}}$ & $46,05^{\mathrm{ns}}$ \\
\hline Resíduo b & 52,61 & 14,14 \\
\hline $\mathrm{CV}_{\exp }(\%)$ & 29,97 & 22,63 \\
\hline Tempo (t) & $9,98^{*}$ & $5,77^{*}$ \\
\hline $\mathrm{t} \times \mathrm{c}$ & $15,31 *$ & $0,31^{\mathrm{ns}}$ \\
\hline $\mathrm{t} \times \mathrm{r}$ & $2,66^{\mathrm{ns}}$ & $0,81^{\mathrm{ns}}$ \\
\hline$t \times c x r$ & $2,17^{\mathrm{ns}}$ & $0,75^{\mathrm{ns}}$ \\
\hline Resíduo c & 1,86 & 0,94 \\
\hline $\mathrm{CV}_{\exp }(\%)$ & 5,63 & 5,84 \\
\hline
\end{tabular}

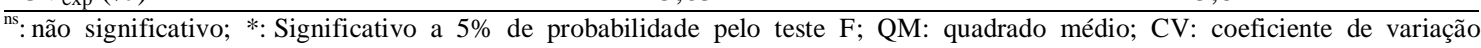
experimental.

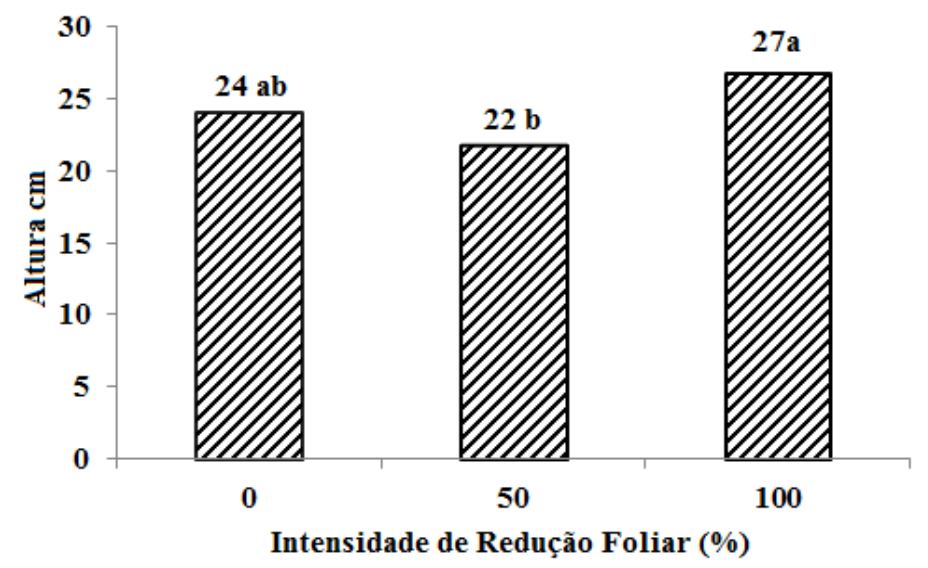

Figura 1. Valores médios de altura de plantas de arnica sob diferentes intensidades de redução foliar. Médias acompanhadas de mesma letra nas barras não diferem ao nível de $95 \%$ de probabilidade pelo teste de Tukey.

Figure 1. Mean values of arnica plant height under different intensities of leaf reduction. Averages followed by the same letter do not differ in bars at $95 \%$ probability by Tukey test.

Outra possível conjectura que se pode fazer é que, devido à espécie Lychnophora pohlii ocorrer em ambientes secos, ela apresenta adaptação à exposição ao sol, à escassez de água e a solos rasos. Assim, ao serem transplantadas para o viveiro, em casa de sombra e com irrigação em abundância, as mudas sofreram inicialmente para se adaptarem, sendo a altura mais afetada do que o diâmetro. Essa reflexão é corroborada pelo fato de que, quando as plantas foram transportadas para céu aberto, onde ocorre a diminuição da irrigação, não foi observado decréscimo delas. O período de permanência sob sombreamento pode ter diminuído a eficiência fotossintética e, consequentemente, a quantidade de fotoassimilados e reguladores de crescimento. Varela e Santos (1992), ao estudarem a influência do sombreamento $(30 \%, 50 \%, 70 \%)$ em mudas de angelim-pedra (Dinizia excelsa), verificaram que as médias da altura e do diâmetro do colo das plantas diminuíram com o aumento do sombreamento. Assim, de acordo com esses autores, a diminuição do crescimento observado com o sombreamento pode ser justificado pela baixa capacidade do sistema fotossintetizante, pois, não havendo produção suficiente de fotoassimilados, o crescimento da planta não é satisfatório. 


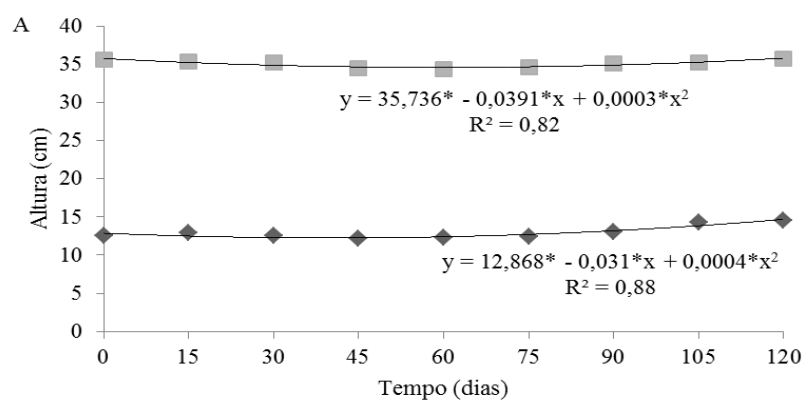

$\downarrow$ Classe I $\square$ Classe II

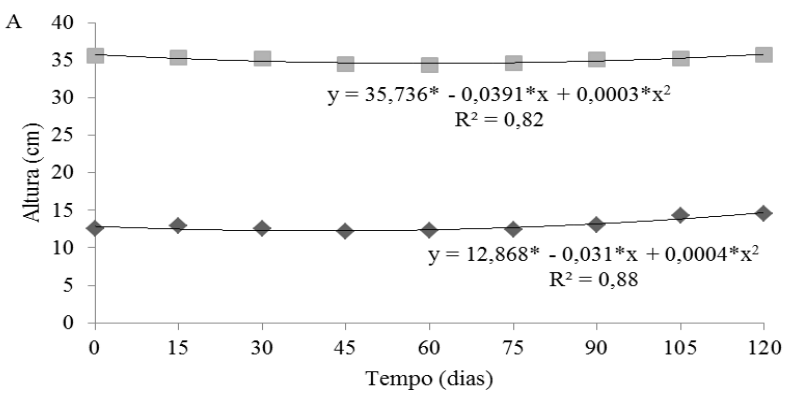

- Classe I Classe II
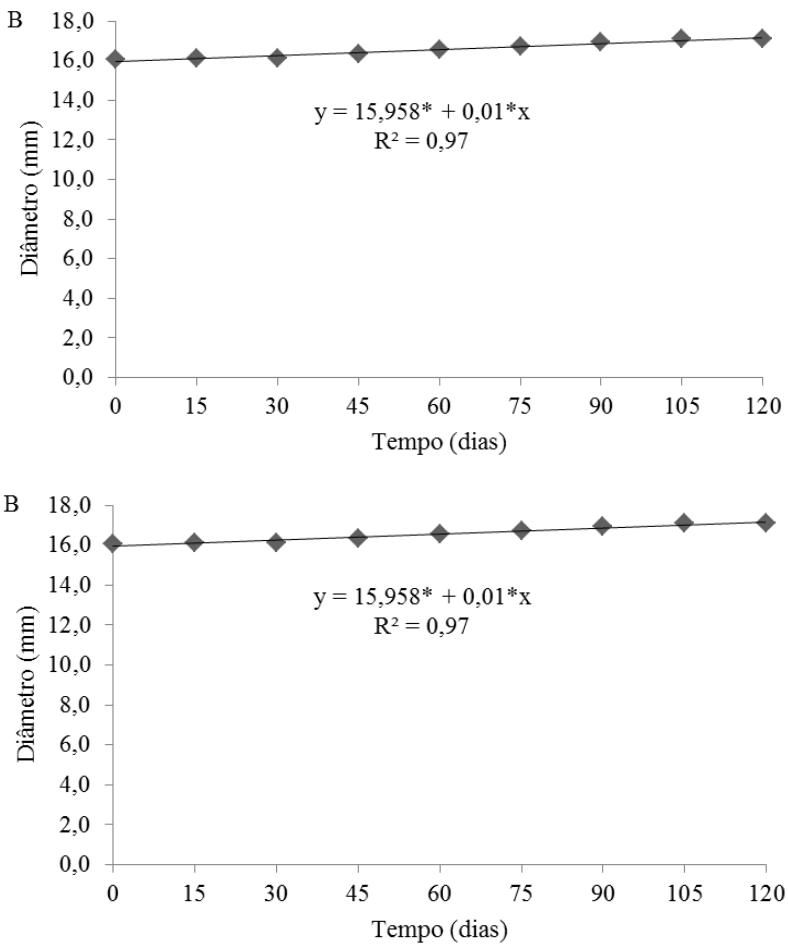

Figura 2. Crescimento em altura para interação tempo x redução foliar (A) e diâmetro em função do tempo classe $\mathrm{x}$ tempo (B), para as mudas de arnica, via resgate. * Significativo a 5\% de probabilidade pelo teste $\mathrm{F}$.

Figure 2. Growth in height to time interaction vs. leaf reduction (A), and diameter class as a function of time vs. time (B), for the arnica seedlings via rescue. * Significant at the $5 \%$ probability level for the $\mathrm{F}$ test.

FLORESTA, Curitiba, PR, v. 45, n. 3, p. 645 - 654, jul. / set. 2015.

Silva, N. F. e. et al.

ISSN eletrônico 1982-4688 / ISSN impresso 0015-3826 
O crescimento incomum em altura apresentado pelas plantas de arnica durante os 60 dias iniciais, pode apresentar estiolamento, previsto para uma espécie "de sol”, ou seja, uma limitação imposta pelo fator de produtividade (luz) intrínseco ao próprio ambiente, assim como a características genéticas da própria espécie, relacionadas a um lento crescimento, ao resultado da composição do substrato utilizado no cultivo das mudas, ou, ainda, à soma desses fatores (GIANOTTI et al., 2013). Segundo Campos e Uchida (2002), são necessários estudos sobre o efeito da composição do substrato, tendo em vista a obtenção de mudas de melhor qualidade e a redução do tempo de permanência no viveiro.

A taxa de sobrevivência dos 240 indivíduos resgatados foi de $49,15 \%$, sendo de $68,3 \%$ para Classe I (2,5 a $20 \mathrm{~cm})$ e de $30 \%$ para Classe II $(25$ a $55 \mathrm{~cm})$. A significância estatística foi observada em nível de interação classes de tamanho x redução foliar e foi acompanhada pelo desdobramento dos graus de liberdade (Tabela 2). Para a Classe I, não foi observada diferença estatística entre as três intensidades de redução foliar, ou seja, diferentes níveis de redução foliar não afetaram a sobrevivência, portanto qualquer uma das intensidades de redução pode ser aplicada. Para a Classe II, a redução de $100 \%$ diferenciou-se das de $0 \%$ e $50 \%$, proporcionando uma maior sobrevivência em relação aos demais tratamentos/intensidades de redução foliar. Ao serem analisadas as classes de altura em função dos tratamentos de redução foliar, observa-se que a Classe I é superior à Classe II. Desse modo, ao fazer o resgate de arnica, recomenda-se coletar plantas de menor altura e não proceder a redução foliar.

Viani e Rodrigues (2007), em estudos sobre resgate de plantas jovens de várias espécies nativas, verificaram maior sobrevivência nas plantas da classe de altura entre 11 e $20 \mathrm{~cm}$, porém evidenciaram que, em todas as classes, os três meses iniciais após o transplante foram o período mais crítico para a sobrevivência das mudas. Calegari et al. (2011), em trabalho semelhante, também encontraram maior sobrevivência em mudas de menor altura.

A alta taxa de mortalidade da arnica pode ser devida ao fato de sua ocorrência estar associada a solos rasos ou entre fendas e afloramentos rochosos, o que torna seu resgate mais difícil, podendo ocasionar danos às raízes. Porém vale ressaltar que o resgate foi realizado em dezembro, em dia chuvoso, sendo as plantas de maior porte mais difíceis de serem coletadas na condição citada anteriormente, o que pode ter acarretado a maior taxa de mortalidade da Classe II.

Tabela 2. Resultados médios da sobrevivência de mudas de arnica em função das três intensidades de redução foliar e das duas classes de tamanho ${ }^{(1)}$.

Table 2. Average results of arnica seedling survival, depending on the three intensities of leaf reduction and two size classes ${ }^{(1)}$.

\begin{tabular}{lccc}
\hline \multirow{2}{*}{ Classes $(\mathbf{c m})$} & \multicolumn{3}{c}{ Redução foliar } \\
\cline { 2 - 4 } & $\mathbf{0 \%}$ & $\mathbf{5 0 \%}$ & $\mathbf{1 0 0 \%}$ \\
\hline $2,5-20$ & $70,00 \mathrm{aA}$ & $72,50 \mathrm{aA}$ & $62,50 \mathrm{aA}$ \\
$25-55$ & $15,00 \mathrm{bB}$ & $27,50 \mathrm{Bb}$ & $47,50 \mathrm{aB}$ \\
\hline
\end{tabular}

Médias seguidas pela mesma letra minúscula na linha e maiúscula na coluna não diferem estatisticamente entre si pelo teste de Tukey a $95 \%$ de probabilidade.

Para verificar a porcentagem de indivíduos que emitiram folhas, foi utilizado um modelo curvilíneo para priorizar o efeito biológico, assim como verificado por Fonseca et al. (2002) ao estudarem o efeito do crescimento de mudas de Trema micranta, que observaram que o número de folhas das mudas aos 120 dias apresentaram efeito quadrático.

Foi observada uma tendência crescente até os 80 dias na porcentagem de indivíduos que emitiram folhas para as curvas referentes às reduções de 100\% (T3 e T6) e 50\% (T5), ocorrendo um decréscimo logo após (Figura 3). Esse fato é explicado por uma capacidade de brotação mais acentuada intrínseca à espécie, pois a poda total das mudas de arnica inibiu a dominância apical (normalmente causado pela maior concentração de auxina). Segundo Cline (1994), a produção de auxina pelas gemas apicais inibe o desenvolvimento de gemas laterais do caule. À medida que ocorre a remoção da gema apical, normalmente, há um estímulo do crescimento das gemas laterais, eliminando a influência desse regulador de crescimento e permitindo que as gemas laterais saiam do estado de dormência e formem ramos, folhas e flores.

Nos tratamentos em que não houve redução foliar (T1 e T4), observou-se um crescimento linear. Segundo Hartmann et al. (2002), para muitas espécies a presença de folhas ou parte delas é uma condição prévia para a produção de auxinas e cofatores que são fontes promotoras de enraizamento. 

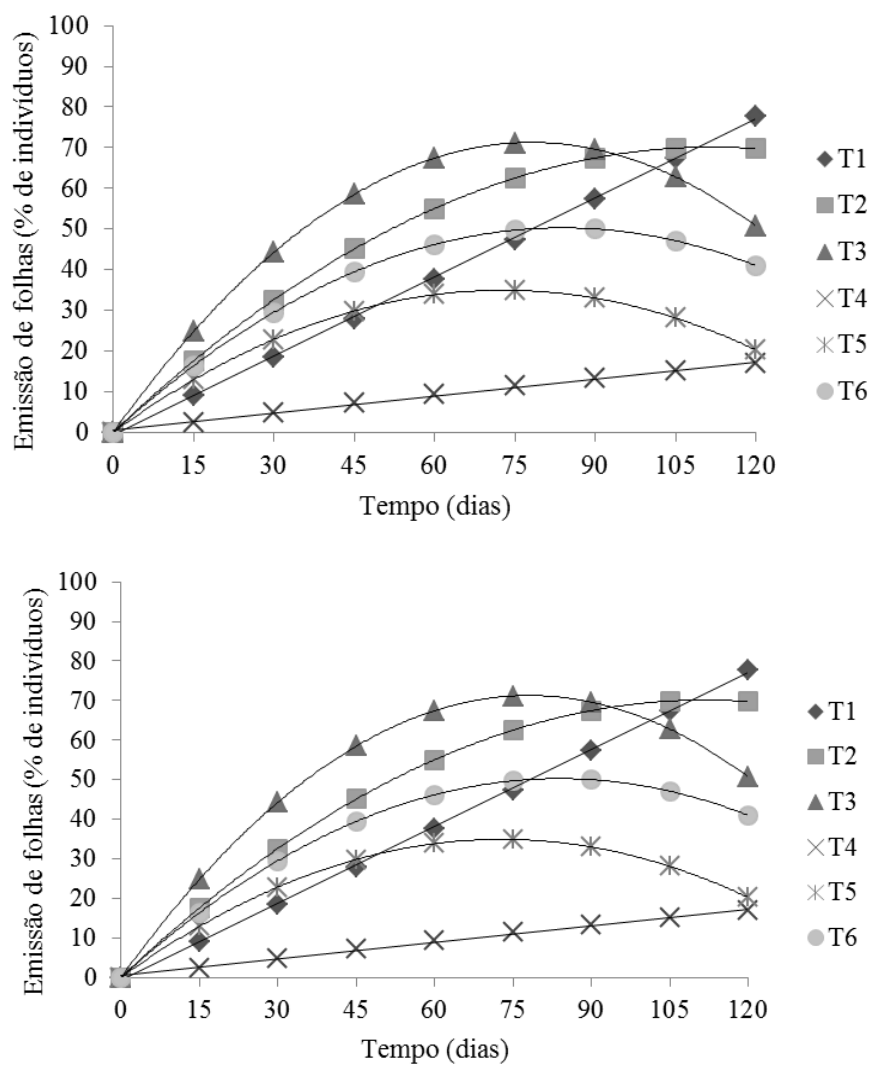

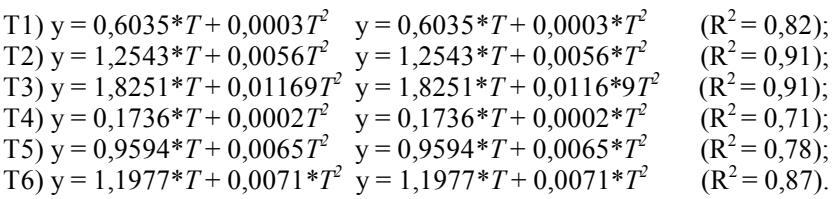

* Significativo a $5 \%$ de probabilidade pelo teste $\mathrm{F}$.

Figura 3. Curvas relacionadas aos ajustes do modelo quadrático $Y=+\beta 2 T e^{x}=\beta 1 T 1+\beta \mathrm{T}^{2}$ para mudas de arnica, resgatadas em função do tempo, em que y: emissão de folhas (\% de indivíduos que emitiram folhas); t: tempo; $\beta_{0} \beta_{0}$ : constante zero. Desse modo: T1 $(0 \%)$, T2 (50\%) e T3 (100\%) são da classe de 2,5 a $20 \mathrm{~cm}$, e T4 (0\%), T5 (50\%) e T6 (100\%) de 25 a $55 \mathrm{~cm}$.

Figure 3. Curves related to the settings of quadratic model $Y=+\beta 2 T e^{x}=\beta 1 T 1+\beta \mathrm{T}^{2}$ for arnica plants, via the redemption function of time, where y: emission leaf (\% of subjects who issued sheets); T: time; $\beta_{0} \beta_{0}$ : constant zero. Thus: T1 $(0 \%), \mathrm{T} 2(50 \%)$ and T3 $(100 \%)$ of the class are from 2.5 to $20 \mathrm{~cm}$, and T4 (0\%), T5 (50\%) and T6 $(100 \%)$ of the 25 to $55 \mathrm{~cm}$.

Vidal (2008), em estudo sobre transplante de plantas, verificou que a família Asteraceae se destacou por apresentar grande emissão de folhas. Viani e Rodrigues (2007) observaram que a porcentagem de indivíduos resgatados que emitiram folhas foi maior até o terceiro mês, diminuindo nas avaliações seguintes, fato que corrobora a maioria dos tratamentos estudados. Fonseca et al. (2002), ao estudarem o efeito do crescimento de mudas de Trema micranta, verificaram que o período de permanência sob sombreamento afetou significativamente o número de folhas aos 120 dias, com ponto mínimo aos 83 dias, porém com posterior aumento no número de folhas das plantas. Aguiar et al. (2005), em estudo com mudas de pau-brasil (Caesalpinia echinata) submetidas a cinco níveis de sombreamento $(0,20,40,60$ e $80 \%)$, observaram a redução do número de folhas com o aumento do sombreamento, apesar da não diferença significativa entre os tratamentos.

FLORESTA, Curitiba, PR, v. 45, n. 3, p. 645 - 654, jul. / set. 2015.

Silva, N. F. e. et al.

ISSN eletrônico 1982-4688 / ISSN impresso 0015-3826

DOI: $10.5380 /$ rf.v45i3.31949 
A técnica de resgate de indivíduos jovens regenerantes é bastante vantajosa e tem sido cada vez mais prescrita como estratégia de conservação, porém ainda é utilizada de forma restrita, quando a destruição do hábitat é iminente (WENDELBERGER et al., 2008). Ressalte-se, no entanto, a importância de investigar tal técnica, sendo recomendada a utilização de procedimentos diferentes para cada espécie ou família botânica. Segundo Santos (2010), a principal barreira ao sucesso do resgate é a carência de metodologias adequadas ao que ecologicamente se requer em operações de restauração de ecossistemas. Assim, para se resgatar indivíduos e espécies com sucesso, sugere-se utilizar diversas formas de coleta de germoplasma e metodologias diversas, para a devida restauração da vegetação suprimida.

O presente estudo demonstrou que o resgate de arnica pode desempenhar um papel importante na conservação da espécie. Assim, nos casos em que a utilização dessa técnica visa o transplante para o viveiro, seria interessante o estudo por espécie, para obtenção de maiores informação sobre a produção de mudas.

\section{CONCLUSÕES}

- O resgate da arnica deve ser realizado para a classe de altura de 2,5-20 cm, devido à sua maior taxa de sobrevivência e crescimento em altura e diâmetro.

- Não é necessário fazer redução foliar de plantas de arnica para a classe de altura de $2,5-20 \mathrm{~cm}$ resgatadas visando à produção de mudas.

- Ajustes na técnica de resgate da arnica precisam ser realizados, a fim de maximizar a sobrevivência de mudas dessa espécie.

\section{AGRADECIMENTOS}

À UFVJM, pelo apoio financeiro. Ao Instituto Estadual de Floresta (IEF), pela licença de realização deste trabalho no Parque Estadual do Biribiri (PEB). À FAPEMIG, CAPES e CNPq, pelo aporte financeiro na forma de projetos individuais e bolsas de pós-graduação.

\section{REFERÊNCIAS}

AGUIAR, F. F. A.; KANASHIRO, S.; TAVARES, A. R.; PINTO, M. M.; STANCATO, G. C.; AGUIAR, J. de; NASCIMENTO, T. D. R. do. Germinação de sementes e formação de mudas de Caesalpinia echinata Lam. (pau-brasil): efeito de sombreamento. Revista Árvore, v. 29, n. 6, p. 871 875, 2005.

CALEGARI, L.; MARTINS, S. V.; BUSATO, L. C.; SILVA, E.; JÚNIOR, R. C.; GLERIANI, J. M. Produção de mudas de espécies arbóreas nativas em viveiro via resgate de plantas jovens. Revista Árvore, v. 35, n. 1, p. 41 - 50, 2011.

CAMPOS, M. A. A.; UCHIDA, T. Influência do sombreamento no crescimento de mudas de três espécies amazônicas. Pesquisa Agropecuária Brasileira, v. 37, n. 3, p. 281 - 288, 2002.

CARNEIRO, J. G. A. Produção e controle de qualidade de mudas florestais. Curitiba: UFPR, 1995. $451 \mathrm{p}$.

CLINE, M. G. The role of hormones in apical dominance. New approaches to an old problem in plant development. Physiologia Plantarum, v. 90, n. 1, p. 230 - 237, 1994.

FONSECA, E. P.; VALÉRI, S. V.; MIGLIORANZA, E.; FONSECA, N. A. N.; COUTO, L. Padrão de qualidade de mudas de Trema micrantha (L.) Blume, produzidas sob diferentes períodos de sombreamento. Revista Árvore, v. 26, n. 4, p. 515 - 523, 2002.

GARCÍA-ORTH, X.; MATÍNEZ-RAMOS, M. M. Isolated trees and grass removal improve performance of transplanted Trema micrantha (L.) Blume (Ulmaceae) saplings in tropical pastures. Restoration Ecology, v. 19, n. 1, p. 24 - 34, 2011.

GIANOTTI, A. R. da C.; SOUZA, M. J. H. de; PEREIRA, I. M.; MACHADO, E. L. M.; VIEIRA, A. D.; MAGALHÃES, M. R. Soil and phytosociological characterization of an area with predominance of arnica (Lychnophora pohlii Sch. Bip.). Revista Brasileira de Ciência do Solo, v. 37, n. 3, 2013. 
GRAEL, C. F. F.; ALBUQUERQUE, S.; LOPES, J. L. C. Chemical constituents of Lychnophora pohlii and trypanocidal activity of crude plant extracts and of isolated compounds. Fitoterapia, v. 76, p. 73 - 82, 2005.

HARTMANN, H. T.; KESTER, D. E.; DAVIES JUNIOR, F. T.; GENEVE, R. L. Plant propagation: principles and practices. 7. ed. New Jersey: Prentice Hall, 2002. 880 p.

HEIDEN, G.; BARBIERI, R. L.; WASUM, R. A.; SCUR, L.; SARTORI, M. A família Asteraceae em São Mateus do Sul, Paraná. Revista Brasileira de Biociências, Porto Alegre, v. 5, supl. 2, p. 249 - 251, 2007.

HOLL, K. D. Effect of shrubs on tree seedling establishment in an abandoned tropical pasture. Journal of Ecology, v. 90, p.179 - 187, 2002.

KANASHIRO, A.; KABEYA, L. M.; GRAEL, C. F. F.; JORDÃO, C. O.; AZZOLINI, A. N. C. S.; LOPES, J. L. C.; LUCISANO-VALIM, Y. M. Sesquiterpene lactones from Lychnophora pohlii: neutrophil chemiluminescence inhibition and free radical scavenger activity. Journal of Pharmacy and Pharmacology, v. 58, p. 853 - 858, 2006.

LAWRENCE, B. A.; KAYE, T. N. Reintroduction of Castilleja levisecta: Effects of ecological similarity, source population genetics, and habitat quality. Restoration Ecology, v. 19, n. 2, p. 166 - 176, 2011.

JACOBI, C. M.; CARMO, F. F. do; VICENT, R. C. Estudo fitossociológico de uma comunidade vegetal sobre canga como subsídio para a reabilitação de áreas mineradas no Quadrilátero Ferrífero, MG. Revista Árvore, v. 32, n. 2, p. 345 - 353, 2008.

JACOBI, C. M.; CARMO, F. F.; VINCENT, R. C.; STEHMANN, J. R. Plant communities on ironstone outcrops: a diverse and endangered Brazilian ecosystem. Biodiversity and Conservation, v. 16, n. 7, p. 2185 - 2200, 2007.

MARTINELLI, G.; MORAES, M. A. 2013. Livro vermelho da flora do Brasil. Jardim Botânico do Rio de Janeiro. <http://cncflora.jbrj.gov.br>. Acesso em: 20/11/2014.

MARTÍNEZ-GARZA, C.; PEÑA, V.; RICKER, M.; CAMPOS, A.; HOWE, H. F. Restoring tropical biodiversity: leaf traits predict growth and survival of late-successional trees in early-successional environments. Forest Ecology and Management, v. 217, n. 2/3, p. 365 - 379, 2005.

MELO, P. R. B.; OLIVEIRA, J. A.; PINTO, J. E. B. P.; CASTRO, E. M.; VIEIRA, A. R.; EVANGELISTA, J. R. E. Germinação de aquênios de arnica (Lychnophora pinaster Mart.) armazenados em diferentes condições. Ciência e Agrotecnologia, v. 31, n. 1, p. 75 - 82, 2007.

NEVES, S. C.; ABREU, P. A. A.; FRAGA, L. M. S. Fisiografia. In SILVA, A. C.; PEDREIRA, L. C. V. S. F.; ABREU, P. A. A. (Eds.). Serra do Espinhaço Meridional: paisagens e ambientes. Belo Horizonte: O Lutador, 2005. p. 47 - 58.

RIBEIRO, T. M.; MARTINS, S. V.; LANA, V. M.; SILVA, K. de A. Sobrevivência e crescimento inicial de plântulas de Euterpe edulis Mart. transplantadas para clareiras e sub-bosque em uma Floresta Estacional Semidecidual, em Viçosa, MG. Revista Árvore, v. 35, n. 6, p. 1219 - 1226, 2011.

RODRIGUES, V. E. G.; CARVALHO, D. A. Levantamento etnobotânico de plantas medicinais no domínio do cerrado na região do alto Rio Grande, Minas Gerais. Ciência e Agrotecnologia, v. 25, n. 1, p. $21-28,2001$.

SANTANA, R. C.; DUTRA, T. R.; CARVALHO NETO, J. P.; NOGUEIRA, G. S.; GRAZZIOTTI, P. H.; BARROS FILHO, N. F. de. Influence of leaf area reduction on clonal production of eucalyptus seedlings. Cerne, v.16, p. 251 - 257, 2010.

SANTOS, L. M. dos. Restauração de campos ferruginosos mediante resgate de flora e uso de topsoil no Quadrilátero Ferrífero, Minas Gerais. 2010. 182 p. Tese (Doutorado em Biologia Vegetal) Instituto de Ciências Biológicas, Universidade Federal de Minas Gerais, Belo Horizonte, 2010.

SOUZA, A. V.; PINTO, J. E. B. P.; BERTOLUCCI, S. K. V.; CORRÊA, R. M.; CASTRO, E. M. Germinação de embriões e multiplicação in vitro de Lychnophora pinaster Mart. Ciências e Agrotecnologia, Edição especial, p. 1532 - 1538, 2003.

FLORESTA, Curitiba, PR, v. 45, n. 3, p. 645 - 654, jul. / set. 2015.

Silva, N. F. e. et al.

ISSN eletrônico 1982-4688 / ISSN impresso 0015-3826

653

DOI: $10.5380 /$ rf.v45i3.31949 
STATSOFT, INC. (2010). STATISTICA (data analysis software system), version 10. <www.statsoft.com>.

VARELA, V. P.; SANTOS, J. dos. Influência do sombreamento na produção de mudas de angelim-pedra (Dinizia excelsa Ducke). Acta Amazonica, v. 22, n. 3, p. 407 - 411, 1992.

VENDRUSCOLO, G. S.; MENTZ, L. A. Estudo da concordância das citações de uso e importância das espécies e famílias utilizadas como medicinais pela comunidade do bairro Ponta Grossa, Porto Alegre, RS, Brasil. Acta Botânica Brasileira, v. 20, n. 2, p. 367 - 382, 2006.

VIANI, R. A. G.; RODRIGUES, R. R. Sobrevivência em viveiro de mudas de espécies nativas retiradas da regeneração natural de remanescente florestal. Pesquisa Agropecuária Brasileira, v. 42, n. 8, p. 1067 - 1075, 2007.

VIDAL, C. Y. Transplante de plântulas e plantas jovens como estratégia de produção de mudas para a restauração de áreas degradadas. 2008. 162 p. Dissertação (Mestrado em Recursos Florestais) Escola Superior de Agricultura Luiz de Queiroz, Piracicaba, 2008.

WENDELBERGER, K. S.; FELLOWS, M. Q. N.; MASCHINSKI, J. Rescue and restoration: experimental translocation of Amorpha herbacea Walter var. crenulata (Rybd.) Isley into a novel urban habitat. Restoration Ecology, v. 16, n. 4, p. 542 - 552, 2008. 